\title{
80 members of the feminist review collective - past and present
}

\begin{tabular}{|c|c|c|}
\hline Alison Light & Katherine Gieve & Ann Curthoys \\
\hline Alison Read & Kerry Schott & Catherine Davies \\
\hline Amal Treacher & Kum-Kum Bhavnani & Clare Alexander \\
\hline An Dekker & Lesley Caldwell & Eleonore Kofman \\
\hline Angela Weir & Lola Young & Emily Banks \\
\hline Ann Phoenix & Loretta Loach & Gail Lewis \\
\hline Ann Wickham & Lorraine Gamman & Hala Shukralla \\
\hline Annette Kuhn & Lucy Bland & Helen Irving \\
\hline Annie $\varepsilon$. Coombes & Lyn Thomas & Jane Haggis \\
\hline Annie Whitehead & Lynne Segal & Jennie Martin \\
\hline AnnMarie Wolpe & Mandy Snell & Mary Hickman \\
\hline Avtar Brah & Mary Mclntosh & Meera Kosambi \\
\hline Caroline Osborne & Maxine Molyneux & Parvati Raghuram \\
\hline Catherine Hall & Merl Storr & Pratibha Parmar \\
\hline Charlotte Brundson & Mica Nava & Valerie Amos \\
\hline Clara Connolly & Michèle Barrett & Wendy Rickard \\
\hline Clara Mulhern & Naila Kabeer & Zarina Maharaj \\
\hline Clare Hemmings & Nel Druce & \\
\hline Cora Kaplan & Nirmal Puwar & Corresponding \\
\hline Delia Jarrett-Macauley & Pam Alldred & \\
\hline Dot Griffiths & Pam Dub & Editors \\
\hline Eileen Phillips & Razia Azia & Ailbhe Smyth \\
\hline Elizabeth Wilson & Rebecca O'Rourke & Ann Curthoys \\
\hline Erica Carter & Rita Rupal & AnnMarie Wolpe \\
\hline Esther Saraga & Sadhana Sutar & Deborah Kasente \\
\hline Felicity Edholm & Sue O'Sullivan & Firdous Azim \\
\hline Gail Lewis & Sonja Ruehl & Gulsum Baydar Nalbantolglu \\
\hline Hannah Kanter & Sharon Morris & Hala Shukrallah \\
\hline Helen Crowley & Sue Himmelweit & Jacqui Alexander \\
\hline Inge Blackman & Sue Readman & Kamala Kempadoo \\
\hline Irene Gedalof & Vicki Bertram & Kum-Kum Bhavnani \\
\hline Jacqueline Andall & Victoria Greenwood & Lidia Curti \\
\hline Jan Brown & Wendy Hollway & Meera Kosambi \\
\hline Jane Parkin & & Patricia Mohammed \\
\hline Judy Keiner & & Sue 0’Sullivan \\
\hline Julia Naish & Guest ec & Vesna Nikolić-Ristanović \\
\hline Karen Margolis & Ailbhe Smythe & \\
\hline Karen Payne & Amina Mama & \\
\hline
\end{tabular}

